\title{
Patterns of Electronic Portal Use among Vulnerable Patients in a Nationwide Practice-based Research Network: From the OCHIN Practice-based Research Network (PBRN)
}

\author{
Lorraine S. Wallace, PhD, Heather Angier, MPH, Nathalie Huguet, PhD, \\ James A. Gaudino, MD, MPH, MS, Alex Krist, MPH, Marla Dearing, MPH, \\ Marie Killerby, MPH, Miguel Marino, PhD, and Jennifer E. DeVoe, MD, DPhil
}

Background: Underserved patient populations experience barriers to accessing and engaging within the complex health care system. Electronic patient portals have been proposed as a potential new way to improve access and engagement. We studied patient portal use for 12 consecutive months (365 days) among a large, nationally distributed, underserved patient population within the OCHIN (originally created as the Oregon Community Health Information Network and renamed OCHIN as other states joined) practice-based research network (PBRN).

Methods: We retrospectively assessed adoption and use of Epic's MyChart patient portal in the first 12 months after MyChart was made available to the OCHIN PBRN. We examined electronic health record data from 36,549 patients aged $\geq 18$ years who were offered a MyChart access code between May 1, 2012, and April 30, 2013, across the OCHIN PBRN in 13 states.

Results: Overall, $29 \%$ of patients offered an access code logged into their MyChart account. Superusers (minimum of 2 logins per month over a 12-month period) accounted for $6 \%$ of users overall. Men, nonwhite patients, Hispanic patients, Spanish-speaking patients, and those with the lowest incomes were significantly less likely to activate. Publicly insured and uninsured patients were also less likely to log in to their MyChart account, but once activated they were more likely than privately insured patients to use MyChart functions.

Conclusions: Our findings suggest that, compared with others, certain patient groups may be less interested in using patient portals or may have experienced significant barriers that prevented use. Making portal access available is a first step. Additional studies need to specifically identify health system-, clinic-, and patient-level barriers and facilitators to portal adoption and use. ( $\mathrm{J}$ Am Board Fam Med 2016;29:592-603.)

Keywords: Ambulatory Care Facilities, Electronic Health Records, Hispanic Americans, Information Services, Medical Assistance, Medically Uninsured, Oregon, Poverty, Practice-based Research, Retrospective Studies, Vulnerable Populations

Underserved patient populations experience barriers to accessing and engaging within the complex health care system, and there are significant disparities in access to care and health outcomes across

This article was externally peer reviewed.

Submitted 28 January 2016; revised 3 May 2016; accepted 9 May 2016.

From the Department of Biomedical Education and Anatomy, The Ohio State University, Columbus, OH (LSW); the Oregon Health \& Science University (HA, NH, JAG, MK, MM, JED); the Virginia Commonwealth University (AK); and OCHIN, Portland, OR (MD). the United States. Widespread use of electronic health records (EHRs) and other technologies now offer new opportunities for patients to become

Funding: Support for this study was provided by the $\mathrm{Na}$ tional Cancer Institute (grant R01 CA181452 and R01 CA168795) and the Patient-Centered Outcomes Research Institute (grant CDRN-1306-04716).

Conflict of interest: none declared.

Corresponding author: Lorraine S. Wallace, PhD, Department of Biomedical Education and Anatomy, The Ohio State University, 2230 North High St, Columbus, OH 43201 (E-mail: Lorraine.Wallace@osumc.edu). 
more active participants in their health care. $\mathrm{Pa}$ tient engagement technologies, such as patient portals, have been proposed as 1 viable solution to overcome health disparities. ${ }^{1}$ Portals allow patients to securely perform a wide range of tasks, including retrieving laboratory results, scheduling appointments, renewing prescriptions, and contacting their health care provider to ask questions. As practices strive to meet national standards for transforming health care and meaningful use benchmarks, ${ }^{2}$ an increasing number are offering patient portals integrated into their EHR. There is a need to understand better the evolving patient portal landscape and whether portals are reaching underserved populations as has been hoped.

To date, a handful of studies have explored various aspects of patient portal use. Collectively, studies across varying geographic regions have reported differences in use of and access to patient portal systems. ${ }^{3-7}$ Most early studies describing patient portal users focused on patient populations with chronic health problems. ${ }^{8-11}$ This previous research identified certain sociodemographics associated with a higher likelihood of use, including young age, white race, female sex, and advanced levels of education. A few studies specific to underserved patients have suggested that underserved populations may be struggling to access these electronic patient engagement resources. For example, in a large cohort of underserved patients in New York City, Ancker et $\mathrm{al}^{3}$ found significant racial and ethnic disparities among patients both receiving an access activation code and using electronic patient portals. However, other studies using focus groups with underserved patient populations ${ }^{12,13}$ reported more encouraging results regarding interest in and acceptance of using personal health portals. Most previous research has been limited to examining electronic portal activation and/or overall use without assessing patterns of use, frequency of use, or types of activities performed while using the portal over time. ${ }^{4-7,14}$ It is likely that patients who do not $\log$ into and use their personal health portal shortly after receiving an access code will not use this service. Thus adoption of the electronic portal immediately after activation is most likely essential for sustainable and continuous utilization of electronic health portals. The purpose of this study was to characterize longitudinal portal use during the first 12 consecutive months (365 days) after initial access to MyChart among a large, underserved adult pa- tient population receiving care at clinics within the OCHIN practice-based research network (PBRN).

\section{Methods}

\section{Data Source and Study Population}

We created an EHR data set that linked data from all OCHIN PBRN practices. OCHIN was originally created as the Oregon Community Health Information Network and renamed OCHIN as other states joined. ${ }^{15,16}$ All OCHIN member clinics, organized and managed within unique service areas, share a centrally hosted, linked instance of the EpicCare EHR. Practices in the OCHIN network installed Epic's English-language personal health record MyChart starting in 2010 (Epic Care and MyChart; Epic Systems, Verona, Wisconsin).

To be included in the analysis, a patient had to be aged $\geq 18$ years and offered a MyChart access code for the first time between May 1, 2012, and April 30, 2013. This time period was selected to include the 12 months just before MyChart codes became automatically provided to all patients. During this 12-month period, leaders at each clinic system were responsible for deciding when and how MyChart was offered to patients, thus creating a "natural experiment." At the time of the study, MyChart was not available in Spanish.

\section{Study Variables}

Because we were interested in the early adoption and use of MyChart, our analyses were limited to the first 12 months (365 days) after initial access to MyChart. Both OCHIN's EHR and MyChart were queried to generate data for this study. The MyChart database was queried to identify patients who were offered an activation code. To capture and characterize patient MyChart log-on frequency over 12 months, 4 categories were constructed: never, once, 2 to 23 times, and $\geq 24$ times. "Superusers" were defined as logging into MyChart a minimum of 24 times (an average of 2 times/ month) over a 12 -month period. ${ }^{17}$

Patient-initiated MyChart activities among those who logged into their accounts were categorized as follows: (1) viewing, (2) online requests or services, and (3) communication. Viewing actions included viewing test results, medications, allergies, current health issues, immunizations, preventive care, medical history, conditions, letters, medical visit summaries and upcoming appointments, and printing a 
wallet card. Online requests or services included completing any of the following activities: requesting a refill, requesting or canceling an appointment, updating sociodemographic data, and downloading a summary record. Communication actions included completing either of the following: communicating with health care team or asking questions regarding prescriptions, medical tests, visit followup, or other nonurgent medical queries.

The following patient sociodemographic characteristics (at the time of receiving initial MyChart access code) were extracted from OCHIN's EHR: sex, age, federal poverty level (FPL), race/ethnicity, preferred language, number of primary care visits, health insurance type, and chronic disease status. We documented formal diagnosis of the following chronic conditions: myocardial infarction, congestive heart disease, peripheral vascular disease, cerebrovascular disease, dementia, chronic pulmonary disease, rheumatic disease, peptic ulcer disease, mild liver disease, diabetes with and without chronic complications, hemiplegia or paraplegia, renal disease, malignancy/cancer, moderate or severe liver disease, metastatic solid tumor carcinoma, and human immunodeficiency virus. Chronic disease frequency was stratified as follows: 0,1 , or $\geq 2$.

\section{Data Analysis}

Differences in patient sociodemographic characteristics across the 4 log-in categories (never, once, 2-23 times, $\geq 24$ times) were initially tested using $\chi^{2}$ tests. To model the association of patient sociodemographic characteristics across the 4 frequency of log-in categories, we used a maximum likelihood multinomial logit regression model, ${ }^{18}$ with "never logged in" as the reference group. This type of regression technique models a 4-level categorical outcome assuming proportional odds and produces risk ratio measures of association in place of odds ratios. All patient sociodemographic characteristics were included as covariates in the models. Clustering of patients within service areas was accounted for using robust clustered sandwich estimators of variance ${ }^{19}$ to estimate risk ratios and accompanying $95 \%$ confidence intervals.

To estimate the association of patients' sociodemographic characteristics with patient-initiated portal activities, adjusted incidence rate ratios were calculated using separate count, zero-inflated Poisson regression models for each of the 3 portal activities as a function of patients' characteristics.
The Vuong ${ }^{20}$ test indicated that the zero-inflated Poisson regression models provided a better fit to the data than standard Poisson regression for each patient-initiated MyChart activity because of the number of excess zeros and data dispersion. Separate zero-inflated Poisson models with all patient characteristics included as covariates were created for each of the 3 MyChart activities, and each accounted for clustering of patients within service areas through robust clustered sandwich estimators. Statistical analyses were performed using STATA, version 12.1 (StataCorp, College Station, TX) and R version 3.2.1 (The R Foundation; available from https://www.r-project.org/); all statistical tests were 2 -sided, and significance was defined as $P<.05$. This study was approved by the Oregon Health \& Science University's Institutional Review Board.

\section{Results}

\section{Patient Sociodemographic Characteristics across MyChart Log-in Categories}

A total of 36,549 patients from OCHIN PBRN clinics in 13 states (Alaska, California, Indiana, Massachusetts, Minnesota, Montana, North Carolina, Nevada, Ohio, Oregon, Texas, Washington, and Wisconsin) comprised the final sample. As outlined in Table 1, the majority of patients who received a MyChart access code but did not log on were female $(60.9 \%)$, white $(84.9 \%)$, non-Hispanic $(81.9 \%)$, identified English as their preferred language $(88.4 \%)$, and did not have a chronic condition $(59.8 \%)$. Approximately one third of the sample was 50 to 64 years old $(28.2 \%)$, uninsured $(34.2 \%)$, and living on less than $100 \%$ of the FPL $(37.9 \%)$.

Of the 36,549 patients offered a MyChart access code, $71.7 \%$ never logged in, $4.7 \%$ patients logged in once, $17.9 \%$ logged in 2 to 23 times, and $5.7 \%$ patients logged in $\geq 24$ times over the course of the first 12 months. Please see Appendix. The average number of MyChart log-in events among those who logged in once was 16.3 (standard deviation, 29.8 ), the median number of logins was 7 , and the number of logins over the 12-month follow-up ranged from 1 to 1055 (data not shown).

Table 2 displays adjusted relative risks by patient characteristics across the 3 MyChart use categories (logged in once, logged in 2 to 23 times, and logged in $\geq 24$ times). Overall, men, nonwhite patients, 
and Hispanic patients were significantly less likely to $\log$ in once, 2 to 23 times, or $\geq 24$ times than women, white patients, or non-Hispanic patients. Patients carrying public insurance were less likely to $\log$ in than those with private insurance across all MyChart usage categories. Patients with income levels $>100 \%$ of the FPL were more likely to $\log$ in $\geq 1$ time than those below the FPL level. The

Table 1. Baseline Patient Sociodemographic Characteristics of OCHIN Community Network Adults $\geq 18$ Years of Age Who Were Issued a MyChart Access Code

\begin{tabular}{|c|c|c|c|c|}
\hline \multirow[b]{2}{*}{ Sociodemographic characteristics } & \multicolumn{4}{|c|}{ Patient Logins } \\
\hline & Refused or Never Logged in & 1 & $2-23$ & $\geq 24$ \\
\hline Total patients & $26,204(71.7)$ & $1,724(4.7)$ & $6,557(17.9)$ & $2,604(5.7)$ \\
\hline \multicolumn{5}{|l|}{ Sex } \\
\hline Female $^{\dagger}$ & $15,965(60.9)$ & $1,114(64.6)$ & $4,242(64.7)$ & $1,399(67.8)$ \\
\hline Male $^{\ddagger}$ & $10,239(39.1)$ & $610(35.4)$ & $2,315(35.3)$ & $665(32.2)$ \\
\hline \multicolumn{5}{|l|}{ Age (years) } \\
\hline $18-29$ & $5,783(22.1)$ & $342(19.8)$ & $1,147(17.5)$ & $336(16.3)$ \\
\hline $30-39$ & $4,721(18.0)$ & $345(20.0)$ & $1,231(18.8)$ & $377(18.3)$ \\
\hline $40-49$ & $4,616(17.6)$ & $341(19.8)$ & $1,170(17.8)$ & $348(16.9)$ \\
\hline $50-64$ & $7,391(28.2)$ & $499(28.9)$ & $2,069(31.6)$ & $709(34.4)$ \\
\hline $65-74$ & $2,361(9.0)$ & $149(8.6)$ & $737(11.2)$ & $229(11.1)$ \\
\hline$\geq 75$ & $1,332(5.1)$ & $48(2.8)$ & $203(3.1)$ & $65(3.1)$ \\
\hline \multicolumn{5}{|l|}{ Race } \\
\hline White & $22,241(84.9)$ & $1,554(90.1)$ & $6,066(92.5)$ & $1,960(95)$ \\
\hline Nonwhite & $3,084(11.8)$ & $129(7.5)$ & $361(5.5)$ & $78(3.8)$ \\
\hline Unknown & $879(3.4)$ & $41(2.4)$ & $130(2.0)$ & $26(1.3)$ \\
\hline \multicolumn{5}{|l|}{ Ethnicity } \\
\hline Non-Hispanic & $21,451(81.9)$ & $1,557(90.3)$ & $6,045(92.2)$ & $1,930(93.5)$ \\
\hline Hispanic & $3,825(14.6)$ & $114(6.6)$ & $369(5.6)$ & $98(4.7)$ \\
\hline Unknown & $928(3.5)$ & $53(3.1)$ & $143(2.2)$ & $36(1.7)$ \\
\hline \multicolumn{5}{|l|}{ Health Insurance } \\
\hline Private & $6,035(23)$ & $586(34)$ & $2,283(34.8)$ & $506(24.5)$ \\
\hline Public & $11,120(42.4)$ & $590(34.2)$ & $2,248(34.3)$ & $945(45.8)$ \\
\hline Uninsured & $8,973(34.2)$ & $542(31.4)$ & $2015(30.7)$ & $612(29.7)$ \\
\hline Unknown & $76(0.3)$ & $6(0.3)$ & $11(0.2)$ & $1(0.0)$ \\
\hline \multicolumn{5}{|c|}{ Number of primary care visits during study period } \\
\hline 0 & $1,286(4.9)$ & $62(3.6)$ & $148(2.3)$ & $5(0.2)$ \\
\hline 1 & $5,615(21.4)$ & $301(17.5)$ & $888(13.5)$ & $55(2.7)$ \\
\hline 2 & $4,317(16.5)$ & $317(18.4)$ & $1,230(18.8)$ & $143(6.9)$ \\
\hline 3 or 4 & $5,836(22.3)$ & $410(23.8)$ & $1,834(28.0)$ & $406(19.7)$ \\
\hline $5-8$ & $5,569(21.3)$ & $374(21.7)$ & $1,614(24.6)$ & $744(36.0)$ \\
\hline$>8$ & $3,581(13.7)$ & $260(15.1)$ & $843(12.9)$ & $711(34.4)$ \\
\hline \multicolumn{5}{|l|}{ Household income } \\
\hline$<100 \% \mathrm{FPL}$ & $9,940(37.9)$ & $529(30.7)$ & $1942(29.6)$ & $724(35.1)$ \\
\hline $100-199 \%$ FPL & $3,624(13.8)$ & $226(13.1)$ & $1042(15.9)$ & $391(18.9)$ \\
\hline 200-399\% FPL & $869(3.3)$ & $115(6.7)$ & $469(7.2)$ & $147(7.1)$ \\
\hline$\geq 400 \% \mathrm{FPL}$ & $2,022(7.7)$ & $176(10.2)$ & $850(13)$ & $224(10.9)$ \\
\hline Unknown & $9,749(37.2)$ & $678(39.3)$ & $2,254(34.4)$ & $578(28.0)$ \\
\hline \multicolumn{5}{|l|}{ Preferred language } \\
\hline English & $23,161(88.4)$ & $1,658(96.2)$ & $6,390(97.5)$ & $2,019(97.8)$ \\
\hline Other & $882(3.4)$ & $39(2.3)$ & $96(1.5)$ & $32(1.6)$ \\
\hline Spanish & $2161(8.2)$ & $27(1.6)$ & $71(1.1)$ & $13(0.6)$ \\
\hline
\end{tabular}




\begin{tabular}{|c|c|c|c|c|}
\hline \multirow[b]{2}{*}{ Sociodemographic characteristics } & \multicolumn{4}{|c|}{ Patient Logins } \\
\hline & Refused or Never Logged in & 1 & $2-23$ & $\geq 24$ \\
\hline \multicolumn{5}{|l|}{ Number of chronic conditions ${ }^{\S}$} \\
\hline 0 & $15,669(59.8)$ & $1,058(61.4)$ & $3,844(58.6)$ & $888(43.0)$ \\
\hline 1 & $6,420(24.5)$ & $441(25.6)$ & $1759(26.8)$ & $664(32.2)$ \\
\hline$\geq 2$ & $4,115(15.7)$ & $225(13.1)$ & $954(14.5)$ & $512(24.8)$ \\
\hline
\end{tabular}

Data are $\mathrm{n}(\%)$.

All comparisons within groups using $\chi^{2}$ test were significant at $P<.001$.

${ }^{\dagger}$ Includes male-to-female transgender patients.

${ }^{\ddagger}$ Includes female-to-male transgender patients.

${ }^{\S}$ Across all entire sample, the most common chronic diseases were chronic pulmonary disease, diabetes without chronic complications, and mild liver disease.

FPL, federal poverty level.

number of visits during the 12 months following receipt of the initial MyChart access code was strongly associated with an increased likelihood of MyChart activation and with more frequent MyChart logins. In fact, adjusted relative risks were well over 3 among patients logging in and $\geq 24$ times, with a suggested "dose response" among these portal users as numbers of clinic visits also increased. Spanish-speaking patients were consistently less likely to $\log$ in once, 2 to 23 times, and $\geq 24$ times compared with English-speaking patients. Though less consistent, speakers of other languages were also less likely to use the portal. After adjusting for other characteristics, the number of chronic conditions was not associated with the likelihood of MyChart log-in frequency, except those with 1 condition were significantly more likely to be superusers ( $\geq 24$ logins).

\section{MyChart Activities}

Table 3 shows the means and ranges of the viewing, online, and communicating activities. Of the 10,345 patients who logged into MyChart at least once, $91.8 \%$ of patients $(\mathrm{n}=9492)$ performed viewing activities (eg, viewing test results, medications, allergies, current action health issues, immunizations, preventive care, medical history, conditions, letters, medical visit summaries and upcoming appointments, and printing a wallet card; median, 16 times for 2-23 MyChart logins and 57 times for $\geq 24$ MyChart logins); $60.9 \%$ of patients $(\mathrm{n}=6296)$ used the request function $(\mathrm{eg}$, requesting a refill, requesting or canceling an appointment, updating sociodemographic data, and downloading a summary record; median, 1 time for
2-23 MyChart logins and 7 times for $\geq 24 \mathrm{My}$ Chart logins); and $58.7 \%$ of patients $(\mathrm{n}=6074)$ used the communicating function (eg, communicating with health care team and asking prescription-related, nonurgent medical-related, medical test-related, or follow-up questions; median, 1 time for 2-23 MyChart logins and 12 times for $\geq 24$ MyChart logins).

Among those with 2 to 23 MyChart logins, the most common viewing activities were viewing test results, followed by viewing medications and viewing health conditions. Similarly, among superusers, the most common viewing activities were viewing test results, followed by viewing medications and viewing upcoming appointments. For both groups (2-23 and $\geq 24$ logins), the most commonly request function activities were requesting or canceling an appointment, requesting a medication refill, and updating demographic information. Communication activities were most frequently used for communicating with health care teams, asking nonurgent medical questions, and asking prescription-related questions for user both groups (2-23 and $\geq 24$ MyChart logins).

Table 4 displays adjusted incidence rate ratios from 3 separate zero-inflated Poisson models examining associations between patients' sociodemographic characteristics and viewing, requesting, and communicating portal activities. Viewing activities were less likely among men than among women. Older patients (age $\geq 65$ years) initiated fewer requesting and viewing activities, whereas patients aged 40 to 74 years used the portal for communicating more than did patients $<40$ years. Both nonwhites and Hispanics had lower rates of com- 
municating activities compared with whites and non-Hispanics, respectively. Publicly insured patients were more likely to use all 3 activities compared with privately insured patients, whereas uninsured patients had higher rates of requesting and viewing activities compared with privately insured patients. Compared with patients with fewer primary care visits during the study period, those with more visits were more likely to initiate requesting and viewing activities. Patients with the highest

Table 2. Adjusted Relative Risk of Using MyChart Once, 2 to 23 Times, and $\geq 24$ Times, by Patient Sociodemographic Characteristics

\begin{tabular}{|c|c|c|c|}
\hline \multirow[b]{2}{*}{ Sociodemographic Characteristics } & \multicolumn{3}{|c|}{ Risk Ratio (95\% Confidence Interval) } \\
\hline & $\begin{array}{l}\text { Logged in Once } \\
\quad(\mathrm{n}=1,724)\end{array}$ & $\begin{array}{l}\text { Logged in } 2-23 \text { Times } \\
\qquad(\mathrm{n}=6,557)\end{array}$ & $\begin{array}{c}\text { Logged in } \geq 24 \text { Times } \\
(\mathrm{n}=2,604)\end{array}$ \\
\hline \multicolumn{4}{|l|}{ Sex } \\
\hline Female* & 1.00 & 1.00 & 1.00 \\
\hline Male $^{+}$ & $0.81(0.69-0.96)$ & $0.81(0.72-0.91)$ & $0.80(0.72-0.90)$ \\
\hline \multicolumn{4}{|l|}{ Age group (years) } \\
\hline $18-29$ & 1.00 & 1.00 & 1.00 \\
\hline $30-39$ & $1.23(1.00-1.53)$ & $1.29(1.16-1.43)$ & $1.21(1.01-1.46)$ \\
\hline $40-49$ & $1.21(0.96-1.54)$ & $1.20(1.05-1.39)$ & $1.01(0.77-1.31)$ \\
\hline $50-64$ & $1.04(0.79-1.36)$ & $1.22(1.04-1.44)$ & $1.09(0.87-1.38)$ \\
\hline $65-74$ & $1.10(0.72-1.68)$ & $1.65(1.21-2.24)$ & $1.17(0.87-1.57)$ \\
\hline$\geq 75$ & $0.64(0.35-1.17)$ & $0.85(0.56-1.27)$ & $0.52(0.34-0.81)$ \\
\hline \multicolumn{4}{|l|}{ Race } \\
\hline White & 1.00 & 1.00 & 1.00 \\
\hline Nonwhite & $0.61(0.38-0.99)$ & $0.45(0.27-0.75)$ & $0.25(0.15-0.43)$ \\
\hline Unknown & $0.93(0.56-1.56)$ & $1.02(0.70-1.48)$ & $0.86(0.57-1.29)$ \\
\hline \multicolumn{4}{|l|}{ Ethnicity } \\
\hline Non-Hispanic & 1.00 & 1.00 & 1.00 \\
\hline Hispanic & $0.68(0.50-0.94)$ & $0.61(0.44-0.84)$ & $0.51(0.35-0.74)$ \\
\hline Unknown & $0.89(0.60-1.30)$ & $0.66(0.53-0.83)$ & $0.71(0.42-1.21)$ \\
\hline \multicolumn{4}{|l|}{ Health insurance } \\
\hline Private & 1.00 & 1.00 & 1.00 \\
\hline Public & $0.64(0.53-0.77)$ & $0.56(0.45-0.70)$ & $0.76(0.60-0.98)$ \\
\hline Uninsured & $0.81(0.66-1.00)$ & $0.80(0.67-0.96)$ & $1.01(0.78-1.31)$ \\
\hline Unknown & $1.33(0.66-2.69)$ & $1.04(0.40-2.67)$ & $4.12(0.36-47.1)$ \\
\hline \multicolumn{4}{|c|}{ Number of primary care visits during study period } \\
\hline 0 & 1.00 & 1.00 & 1.00 \\
\hline 1 & $1.09(0.69-1.72)$ & $1.2(0.79-1.84)$ & $2.60(1.18-5.76)$ \\
\hline 2 & $1.54(0.98-2.42)$ & $2.19(1.42-3.38)$ & $8.78(4.04-19.1)$ \\
\hline 3 or 4 & $1.60(1.02-2.49)$ & $2.60(1.64-4.13)$ & $19.3(9.06-41.1)$ \\
\hline $5-8$ & $1.68(1.07-2.63)$ & $2.64(1.64-4.25)$ & $39.1(18.3-83.5)$ \\
\hline$>8$ & $1.94(1.17-3.20)$ & $2.31(1.34-3.99)$ & $59.4(26.9-131.3)$ \\
\hline \multicolumn{4}{|l|}{ Household income } \\
\hline$<100 \% \mathrm{FPL}$ & 1.00 & 1.00 & 1.00 \\
\hline 100-199\% FPL & $1.05(0.90-1.22)$ & $1.22(1.10-1.36)$ & $1.50(1.34-1.68)$ \\
\hline 200-399\% FPL & $1.90(1.48-2.44)$ & $1.89(1.59-2.23)$ & $2.62(2.05-3.35)$ \\
\hline$\geq 400 \% \mathrm{FPL}$ & $1.39(1.08-1.79)$ & $1.68(1.39-2.02)$ & $1.64(1.29-2.08)$ \\
\hline Unknown & $1.13(0.91-1.41)$ & $0.94(0.70-1.27)$ & $0.94(0.62-1.45)$ \\
\hline \multicolumn{4}{|l|}{ Language } \\
\hline English & 1.00 & 1.00 & 1.00 \\
\hline Other & $0.79(0.48-1.31)$ & $0.60(0.36-0.99)$ & $0.82(0.45-1.48)$ \\
\hline Spanish & $0.23(0.12-0.43)$ & $0.17(0.090-0.31)$ & $0.10(0.038-0.28)$ \\
\hline
\end{tabular}


Table 2. Continued.

\begin{tabular}{|c|c|c|c|}
\hline \multirow[b]{2}{*}{ Sociodemographic Characteristics } & \multicolumn{3}{|c|}{ Risk Ratio (95\% Confidence Interval) } \\
\hline & $\begin{array}{l}\text { Logged in Once } \\
(\mathrm{n}=1,724)\end{array}$ & $\begin{array}{c}\text { Logged in 2-23 Times } \\
(\mathrm{n}=6,557)\end{array}$ & $\begin{array}{c}\text { Logged in } \geq 24 \text { Times } \\
(n=2,604)\end{array}$ \\
\hline \multicolumn{4}{|l|}{ Number of chronic conditions } \\
\hline 0 & 1.00 & 1.00 & 1.00 \\
\hline 1 & $0.99(0.90-1.10)$ & $1.02(0.90-1.16)$ & $1.26(1.07-1.48)$ \\
\hline$\geq 2$ & $0.82(0.64-1.05)$ & $0.86(0.71-1.05)$ & $1.16(0.86-1.57)$ \\
\hline
\end{tabular}

These are risk ratios, not odds ratios. We fit a maximum-likelihood multinomial logit model to estimate probability of (1) having no MyChart use, (2) logging in once on MyChart, or (3) logging in more than once on MyChart. The risk ratio is then the ratio of 2 probabilities conditional on the covariate of interest. This multinomial model included all covariates included in the table, and the risk ratios are all in reference to "no MyChart use." Models accounted for clustering of patients within service areas by including robust clustered sandwich estimators of variance to estimate rate ratio confidence intervals. The bold values are statistically significant at $P<.05$.

*Includes male-to-female transgender patients.

${ }^{\dagger}$ Includes female-to-male transgender patients.

FPL, federal poverty level.

number of primary care visits $(>8)$ during the study period were far more likely to partake in communication-related activities compared with those with fewer primary care visits. Patients with $\geq 1$ chronic condition were also more likely to participate in all 3 activities compared with patients without chronic conditions.

\section{Discussion}

In this study of underserved patients located in 13 states across the United States during an initial patient portal rollout period when participating clinic systems could use various portal recruitment strategies, we found that a majority of patients receiving an access code from their health care provider never attempted to $\log$ in to the MyChart platform. Interestingly, the OCHIN patients studied here were much less likely to activate their MyChart account compared with similar patient populations. For instance, more than two thirds of primary care patients at a large, urban, Midwestern practice activated their electronic patient portal, ${ }^{6}$ whereas $60 \%$ of patients receiving care at federally qualified health centers in the greater New York City area activated their electronic patient portal. ${ }^{3}$

Mirroring previous studies, ${ }^{3,6}$ patients carrying public health insurance (mainly Medicaid or Medicare) or without coverage were less likely to activate MyChart than privately insured patients. While these patients were less likely to activate their MyChart accounts, however, once they logged in they utilized the functionalities of the portal generally more often. Most patients who activated their ac- counts had logged into MyChart more than once, suggesting they found the portal helpful. The findings from this preliminary study characterizing MyChart adoption and use suggest both the need to better understand specific barriers and facilitators experienced by underserved patients in using patient portals and to identify best practices by health systems and providers in recruiting and retaining patients to use portals, such as the importance of encouraging all patients to activate their accounts upon receipt of a portal access code.

Notwithstanding, it is important to recognize known barriers to access to and use of patient portal systems, including limited access to the necessary technology, low functional health literacy, and concerns regarding the privacy and security of personal health information. ${ }^{12,21,22}$ These results point to the potential need for developing, testing, and implementing systematic strategies to facilitate initial activation and use of personal portals by patients. Clinical practices, in partnership with community organizations and technology companies, could perhaps offer electronic portal activation and demonstration workshops to patients, where they could set up their accounts with active support and instruction.

This study is, to our knowledge, the first to report patient characteristics and practices of $\mathrm{My}$ Chart superusers (defined as logging into MyChart a minimum of 24 times [an average of 2 times/ month] over a 12 -month period). ${ }^{14}$ Overall, $29 \%$ of patients logged into MyChart; however, superusers represented $6 \%$ of those who logged in. As could 


\begin{tabular}{|c|c|c|c|}
\hline & \multicolumn{3}{|c|}{ Activity, Mean (Minimum, Maximum) } \\
\hline & Logged in Once & $\begin{array}{c}\text { Logged in 2-23 } \\
\text { Times }\end{array}$ & $\begin{array}{c}\text { Logged in } \geq 24 \\
\text { Times }\end{array}$ \\
\hline \multicolumn{4}{|l|}{ Viewing activities } \\
\hline Test results & $0.44(0,12)$ & $3.17(0,22)$ & $12.30(0,122)$ \\
\hline Medications & $0.53(0,10)$ & $2.58(0,19)$ & $9.51(0,148)$ \\
\hline Allergies & $0.51(0,6)$ & $2.35(0,15)$ & $7.29(0,57)$ \\
\hline Current health issues & $0.49(0,2)$ & $2.14(0,15)$ & $6.01(0,56)$ \\
\hline Print wallet card & $0.03(0,1)$ & $0.14(0,5)$ & $0.52(0,14)$ \\
\hline Immunizations & $0.52(0,6)$ & $2.36(0,15)$ & $7.13(0,56)$ \\
\hline Preventive care & $0.30(0,11)$ & $1.86(0,16)$ & $6.88(0,58)$ \\
\hline Medical history & $0.23(0,2)$ & $0.93(0,13)$ & $2.70(0,55)$ \\
\hline My conditions & $0.52,(0,6)$ & $2.44(0,15)$ & $7.63(0,58)$ \\
\hline Letters & $0.12(0,2)$ & $0.58(0,11)$ & $2.20(0,97)$ \\
\hline After-visit summary & $0.06(0,1)$ & $0.44(0,10)$ & $2.00(0,69)$ \\
\hline Upcoming appointments & $0.13(0,10)$ & $1.32(0,16)$ & $7.68(0,146)$ \\
\hline All viewing activities & $3.89(0,64)$ & $20.30(0,133)$ & $71.86(0,547)$ \\
\hline \multicolumn{4}{|l|}{ Online requests or services } \\
\hline Request a refill & $0.03(0,2)$ & $0.36(0,13)$ & $2.77(0,82)$ \\
\hline Request or cancel an appointment & $0.09(0,3)$ & $1.00(0,14)$ & $5.99(0,96)$ \\
\hline Update demographics (address, phone, E-mail) & $0.03(0,1)$ & $0.19(0,7)$ & $0.75(0,13)$ \\
\hline Download summary record & $0.03(0,1)$ & $0.09(0,4)$ & $0,21(0,12)$ \\
\hline All online activities & $0.19(0,4)$ & $1.64(0,17)$ & $9.73(0,116)$ \\
\hline \multicolumn{4}{|l|}{ Communication activities } \\
\hline Communicating with health care team & $0.14(0,3)$ & $1.34(0,16)$ & $9.46(0,147)$ \\
\hline Nonurgent medical question & $0.05(0,2)$ & $0.64(0,13)$ & $5.31(0,113)$ \\
\hline Prescription question & $0.01(0,2)$ & $0.16(0,8)$ & $1.17(0,23)$ \\
\hline Test results question & $<0.01(0,2)$ & $0.08(0,4)$ & $0.31(0,9)$ \\
\hline Visit follow-up question & $<0.01(0,1)$ & $0.09(0,8)$ & $0.57(0,28)$ \\
\hline All communicating activities & $0.22(0,5)$ & $2.32(0,29)$ & $16.83(0,258)$ \\
\hline
\end{tabular}

be expected, superusers were more likely to view pages within MyChart compared with those logging in on a less frequent basis. Superusers were also much more likely to use the features provided by MyChart, such as communicating with providers and requesting-related activities. To date, few studies have examined the frequency of use of different functions available in patient's electronic portals. ${ }^{4,7}$ Jhamb et $\mathrm{al}^{4}$ found that provider communication among portal users occurred approximately twice a year, a sharp contrast with superusers in our study, suggesting that frequent portal users (superusers) tend to be more actively involved in their health care.

Similar to previous studies, we found that female, white, non-Hispanic, English-speaking, and younger patients were more likely to access and use the MyChart portal. These disparities persisted in each of the log-in frequency groups, but differences were not as prevalent across types of MyChart activities performed, especially among users of different age groups. Few studies have examined the link between health care utilization and use of patient portals. We found a linear relationship of increasing relative risk ratios for logging in as the number of clinic encounters also increased, especially—and not surprisingly-among superusers. Interestingly, and contrary to previous findings, ${ }^{11,23}$ the presence of chronic health conditions was not associated with the likelihood of logging in to MyChart. Compared with those without chronic conditions, however, patients with chronic conditions were more likely to participate in all 3 types of MyChart activities.

These results should be considered in the context of several limitations. First, we did not study the influences of different health system, clinic, and provider factors on portal adoption, use, and reten- 
Table 4. Adjusted Incidence Rates for MyChart Activities by Patient Sociodemographic Characteristics

\begin{tabular}{|c|c|c|c|}
\hline \multirow[b]{2}{*}{ Sociodemographic Characteristics } & \multicolumn{3}{|c|}{ Incidence Rate Ratios (95\% CIs) } \\
\hline & Requesting & Viewing & Communicating \\
\hline \multicolumn{4}{|l|}{ Sex } \\
\hline Female & 1.00 & 1.00 & 1.00 \\
\hline Male & $0.99(0.93-1.05)$ & $0.93(0.88-0.98)$ & $0.97(0.88-1.08)$ \\
\hline \multicolumn{4}{|l|}{ Age group (years) } \\
\hline $18-29$ & 1.00 & 1.00 & 1.00 \\
\hline $30-39$ & $0.98(0.87-1.11)$ & $0.99(0.89-1.10)$ & $1.12(0.97-1.28)$ \\
\hline $40-49$ & $1.00(0.91-1.10)$ & $0.98(0.91-1.07)$ & $1.22(1.11-1.35)$ \\
\hline $50-64$ & $1.03(0.93-1.15)$ & $0.94(0.89-0.99)$ & $1.31(1.15-1.48)$ \\
\hline $65-74$ & $0.78(0.67-0.91)$ & $0.82(0.74-0.90)$ & $1.25(1.04-1.49)$ \\
\hline$\geq 75$ & $0.70(0.57-0.86)$ & $0.79(0.67-0.94)$ & $0.98(0.77-1.25)$ \\
\hline \multicolumn{4}{|l|}{ Race } \\
\hline White & 1.00 & 1.00 & 1.00 \\
\hline Nonwhite & $0.94(0.79-1.11)$ & $0.94(0.81-1.10)$ & $0.69(0.60-0.79)$ \\
\hline Unknown & $0.96(0.82-1.11)$ & $1.05(0.87-1.25)$ & $0.8(0.59-1.09)$ \\
\hline \multicolumn{4}{|l|}{ Ethnicity } \\
\hline Non-Hispanic & 1.00 & 1.00 & 1.00 \\
\hline Hispanic & $1.00(0.88-1.13)$ & $0.97(0.89-1.05)$ & $0.78(0.67-0.91)$ \\
\hline Unknown & $0.68(0.56-0.84)$ & $0.85(0.69-1.05)$ & $1.23(0.88-1.71)$ \\
\hline \multicolumn{4}{|l|}{ Health insurance } \\
\hline Private & 1.00 & 1.00 & 1.00 \\
\hline Public & $1.33(1.20-1.47)$ & $1.16(1.08-1.25)$ & $1.23(1.11-1.35)$ \\
\hline Uninsured & $1.23(1.08-1.40)$ & $1.13(1.05-1.23)$ & $1.13(0.99-1.28)$ \\
\hline Unknown & $2.2(0.81-5.97)$ & $1.77(0.84-3.72)$ & $0.69(0.25-1.87)$ \\
\hline \multicolumn{4}{|c|}{ Number of primary care visits during study period } \\
\hline 0 & 1.00 & 1.00 & 1.00 \\
\hline 1 & $1.35(0.87-2.08)$ & $0.99(0.78-1.25)$ & $0.86(0.37-2.01)$ \\
\hline 2 & $1.69(1.05-2.71)$ & $1.24(0.99-1.54)$ & $1.11(0.48-2.57)$ \\
\hline 3 or 4 & $2.37(1.62-3.46)$ & $1.53(1.26-1.87)$ & $1.33(0.61-2.90)$ \\
\hline $5-8$ & $3.33(2.23-4.97)$ & $2.05(1.66-2.52)$ & $1.87(0.87-4.03)$ \\
\hline$>8$ & $4.91(3.26-7.40)$ & $2.59(2.12-3.17)$ & $2.69(1.22-5.92)$ \\
\hline \multicolumn{4}{|l|}{ Household income } \\
\hline$<100 \% \mathrm{FPL}$ & 1.00 & 1.00 & 1.00 \\
\hline 100-199\% FPL & $1.07(0.98-1.17)$ & $0.99(0.94-1.05)$ & $1.07(0.96-1.19)$ \\
\hline 200-399\% FPL & $1.18(0.97-1.44)$ & $1.02(0.90-1.16)$ & $1.24(1.00-1.53)$ \\
\hline$\geq 400 \% \mathrm{FPL}$ & $1.00(0.89-1.14)$ & $1.04(0.94-1.15)$ & $1.00(0.91-1.10)$ \\
\hline Unknown & $0.96(0.78-1.17)$ & $0.95(0.82-1.10)$ & $0.98(0.79-1.22)$ \\
\hline \multicolumn{4}{|l|}{ Preferred language } \\
\hline English & 1.00 & 1.00 & 1.00 \\
\hline Spanish & $1.03(0.81-1.33)$ & $1.01(0.81-1.26)$ & $0.8(0.56-1.14)$ \\
\hline Other & $1.04(0.82-1.33)$ & $1.09(0.93-1.29)$ & $1.13(0.94-1.36)$ \\
\hline \multicolumn{4}{|l|}{ Number of chronic conditions } \\
\hline 0 & 1.00 & 1.00 & 1.00 \\
\hline 1 & $1.13(1.02-1.26)$ & $1.14(1.08-1.21)$ & $1.09(1.02-1.16)$ \\
\hline$\geq 2$ & $1.19(1.08-1.31)$ & $1.20(1.09-1.32)$ & $1.10(1.00-1.21)$ \\
\hline
\end{tabular}

Only patients who logged into MyChart were included in these models. These are incidence rate ratios, not odds ratios. We fit a separate zero-inflated maximum-likelihood Poisson model to estimate the number of each of the 3 activities. Models accounted for clustering of patients within service areas by including robust clustered sandwich estimators of variance to estimate rate ratio confidence intervals. Bold values are statistically significant at $P<.05$.

CI, confidence interval; FPL, federal poverty level. 
tion. In particular, during this period, a natural experiment of sorts occurred, during which different participating health systems or service areas, clinics, and providers had the option of using different strategies and levels of effort to recruit and retain patient portal users. For example, whereas some clinics may have offered MyChart activation during clinic check-in through support staff or by mail or E-mail, others may have had providers directly recruiting their patients face-to-face or using other methods. While we accounted for patient clustering in service areas to adjust for some of these differences to study the effects of patient characteristics, we did not evaluate system- or provider-level impacts. Second, our findings are based on 1 patient portal, MyChart, and may not be generalizable to other electronic systems or patient populations. However, our findings are likely to be robust for this population since they are based on the study of a large cohort of diverse underserved patients seen at a wide range of safety-net clinics throughout 13 states nationally. Third, though it is clear that portal users were more likely to use MyChart functions if they had chronic diseases, we did not evaluate whether those with more recent chronic disease or other diagnoses might be more likely to use the portal during the initial stages of their diagnostic workups or treatments. Fourth, at the time of the study, the MyChart Spanish-language portal was not available. As a result, the study sample included a small proportion of Spanishspeaking patients.

Despite these limitations, our findings provide important insights into barriers and facilitators of consistent electronic portal use by a diverse group of underserved patients. Future studies need to identify best practices for helping patients better understand the value of using patient portals in managing their health care and to pinpoint patientand care system-level barriers that impede portal use while recognizing that some patients will not or cannot use them. In particular, future studies need to evaluate strategies that further facilitate first use, especially among racial and other minorities, men, older adults, and those who are publicly insured or uninsured, as critical step toward increasing electronic portal use. Finally, as patient portal use continues to increase, additional research is warranted to specify how patient portal use can be further leveraged to improve patient and clinical outcomes and transform health care delivery.

\section{References}

1. Turner-Lee N, Smedley BD, Miller J. Minorities, mobile broadband and the management of chronic diseases. Washington, DC: Joint Center for Political and Economic Studies; 2012.

2. Carayon P, Hoonakker P, Cartmill R, Hassol A. Using health information technology (IT) in practice redesign: impact of health IT on workflow. Patientreported health IT and workflow. Final contract report. AHRQ publication no. 15-0043-EF. Rockville MD: Agency for Healthcare Rsearch and Quality; 2015.

3. Ancker JS, Barron Y, Rockoff ML, et al. Use of an electronic patient portal among disadvantaged populations. J Gen Intern Med 2011;26:1117-23.

4. Jhamb M, Cavanaugh KL, Bian A, et al. Disparities in electronic health record patient portal use in nephrology clinics. Clin J Am Soc Nephrol 2015;10: 2013-22.

5. Mikles SP, Mielenz TJ. Characteristics of electronic patient-provider messaging system utilisation in an urban health care organisation. J Innov Health Inform 2015;22:214-21.

6. Goel MS, Brown TL, Williams A, Hasnain-Wynia $\mathrm{R}$, Thompson JA, Baker DW. Disparities in enrollment and use of an electronic patient portal. J Gen Intern Med 2011;26:1112-6.

7. Smith SG, O'Conor R, Aitken W, Curtis LM, Wolf MS, Goel MS. Disparities in registration and use of an online patient portal among older adults: findings from the LitCog cohort. J Am Med Inform Assoc 2015;22:888-95.

8. Wade-Vuturo AE, Mayberry LS, Osborn CY. Secure messaging and diabetes management: experiences and perspectives of patient portal users. J Am Med Inform Assoc 2013;20:519-25.

9. Riippa I, Linna M, Ronkko I, Kroger V. Use of an electronic patient portal among the chronically ill: an observational study. J Med Internet Res 2014;16: e275.

10. Harrison TG, Wick J, Ahmed SB, et al. Patients with chronic kidney disease and their intent to use electronic personal health records. Can J Kidney Health Dis 2015;2:23.

11. Jones JB, Weiner JP, Shah NR, Stewart WF. The wired patient: patterns of electronic patient portal use among patients with cardiac disease or diabetes. J Med Internet Res 2015;17:e42.

12. Dhanireddy S, Walker J, Reisch L, Oster N, Delbanco T, Elmore JG. The urban underserved: attitudes towards gaining full access to electronic medical records. Health Expect 2014;17:724-32.

13. Zarcadoolas C, Vaughon WL, Czaja SJ, Levy J, Rockoff ML. Consumers' perceptions of patientaccessible electronic medical records. J Med Internet Res 2013;15:e168.

14. Palen TE, Ross C, Powers JD, Xu S. Association of online patient access to clinicians and medical re- 
cords with use of clinical services. JAMA 2012;308: 2012-9.

15. Devoe JE, Gold R, Spofford M, et al. Developing a network of community health centers with a common electronic health record: description of the Safety Net West Practice-based Research Network (SNW-PBRN). J Am Board Fam Med 2011;24:597604.

16. Devoe JE, Sears A. The OCHIN community information network: bringing together community health centers, information technology, and data to support a patient-centered medical village. J Am Board Fam Med 2013;26:271-8.

17. Phelps RG, Taylor J, Simpson K, Samuel J, Turner AN. Patients' continuing use of an online health record: a quantitative evaluation of 14,000 patient years of access data. J Med Internet Res 2014;16: e241.
18. Greene WH. Econometric analysis. 7th ed. Upper Saddle River, NJ: Prentice Hall; 2012.

19. Williams RL. A note on robust variance estimation for cluster-correlated data. Biometrics 2000;56: 645-6.

20. Vuong QH. Likelihood ratio tests for model selection and nonnested hypotheses. Econometrica 1989; 57:307-33.

21. Goel MS, Brown TL, Williams A, Cooper AJ, Hasnain-Wynia R, Baker DW. Patient reported barriers to enrolling in a patient portal. J Am Med Inform Assoc 2011;18(Suppl 1):i8-12.

22. Kruse CS, Argueta DA, Lopez L, Nair A. Patient and provider attitudes toward the use of patient portals for the management of chronic disease: a systematic review. J Med Internet Res 2015;17:e40.

23. Weingart SN, Rind D, Tofias Z, Sands DZ. Who uses the patient Internet portal? The PatientSite experience. J Am Med Inform Assoc 2006;13:91-5. 
Patient Sociodemographic Characteristics Associated with Odds of Not Performing Each MyChart Activity

\begin{tabular}{|c|c|c|c|}
\hline \multirow[b]{2}{*}{ Sociodemographic Characteristics } & \multicolumn{3}{|c|}{ Odds Ratio (95\% Confidence Interval) } \\
\hline & Requesting & Viewing & Communicating \\
\hline \multicolumn{4}{|l|}{ Sex } \\
\hline Female & 1.00 & 1.00 & 1.00 \\
\hline Male & $1.21(1.10-1.32)$ & $1.24(1.12-1.38)$ & $1.22(1.12-1.34)$ \\
\hline \multicolumn{4}{|l|}{ Age group (years) } \\
\hline $18-29$ & 1.00 & 1.00 & 1.00 \\
\hline $30-39$ & $0.81(0.74-0.88)$ & $0.80(0.73-0.88)$ & $0.74(0.67-0.83)$ \\
\hline $40-49$ & $0.87(0.77-0.99)$ & $0.87(0.77-0.99)$ & $0.81(0.71-0.93)$ \\
\hline $50-64$ & $0.86(0.74-0.99)$ & $0.86(0.73-1.01)$ & $0.78(0.68-0.91)$ \\
\hline $65-74$ & $0.72(0.58-0.88)$ & $0.69(0.53-0.90)$ & $0.68(0.53-0.88)$ \\
\hline$\geq 75$ & $1.34(0.98-1.83)$ & $1.35(0.93-1.94)$ & $1.29(0.90-1.84)$ \\
\hline \multicolumn{4}{|l|}{ Race } \\
\hline White & 1.00 & 1.00 & 1.00 \\
\hline Nonwhite & $2.37(1.47-3.83)$ & $2.40(1.51-3.81)$ & $2.41(1.46-3.98)$ \\
\hline Unknown & $1.07(0.75-1.53)$ & $1.02(0.75-1.39)$ & $1.18(0.87-1.60)$ \\
\hline \multicolumn{4}{|l|}{ Ethnicity } \\
\hline Non-Hispanic & 1.00 & 1.00 & 1.00 \\
\hline Hispanic & $1.57(1.15-2.14)$ & $1.65(1.23-2.21)$ & $1.63(1.22-2.16)$ \\
\hline Unknown & $1.30(1.07-1.57)$ & $1.47(1.21-1.79)$ & $1.35(1.13-1.61)$ \\
\hline \multicolumn{4}{|l|}{ Health insurance } \\
\hline Private & 1.00 & 1.00 & 1.00 \\
\hline Public & $1.48(1.28-1.71)$ & $1.60(1.30-1.96)$ & $1.54(1.28-1.86)$ \\
\hline Uninsured & $1.13(0.98-1.31)$ & $1.19(1.00-1.42)$ & $1.15(0.99-1.34)$ \\
\hline Unknown & $1.03(0.40-2.71)$ & $0.99(0.43-2.30)$ & $0.34(0.15-0.77)$ \\
\hline \multicolumn{4}{|c|}{ Number of primary care visits during study period } \\
\hline 0 & 1.00 & 1.00 & 1.00 \\
\hline 1 & $1.03(0.67-1.56)$ & $0.85(0.57-1.26)$ & $0.57(0.41-0.79)$ \\
\hline 2 & $0.49(0.34-0.71)$ & $0.48(0.33-0.69)$ & $0.32(0.22-0.46)$ \\
\hline 3 or 4 & $0.36(0.25-0.53)$ & $0.39(0.26-0.57)$ & $0.24(0.17-0.35)$ \\
\hline $5-8$ & $0.28(0.19-0.40)$ & $0.33(0.23-0.49)$ & $0.20(0.14-0.28)$ \\
\hline$>8$ & $0.24(0.16-0.37)$ & $0.31(0.20-0.48)$ & $0.17(0.11-0.27)$ \\
\hline \multicolumn{4}{|l|}{ Starting household income } \\
\hline$<100 \% \mathrm{FPL}$ & 1.00 & 1.00 & 1.00 \\
\hline 100-199\% FPL & $0.82(0.73-0.92)$ & $0.79(0.73-0.86)$ & $0.77(0.70-0.84)$ \\
\hline 200-399\% FPL & $0.63(0.53-0.75)$ & $0.51(0.43-0.59)$ & $0.58(0.49-0.67)$ \\
\hline$\geq 400 \% \mathrm{FPL}$ & $0.66(0.55-0.80)$ & $0.63(0.53-0.75)$ & $0.65(0.54-0.78)$ \\
\hline Unknown & $1.09(0.84-1.41)$ & $1.05(0.78-1.42)$ & $1.13(0.80-1.58)$ \\
\hline \multicolumn{4}{|l|}{ Preferred language } \\
\hline English & 1.00 & 1.00 & 1.00 \\
\hline Spanish & $1.65(1.06-2.55)$ & $1.47(0.87-2.46)$ & $1.69(1.07-2.67)$ \\
\hline Other & $6.17(3.35-11.4)$ & $6.07(3.35-11.0)$ & $5.74(2.96-11.1)$ \\
\hline \multicolumn{4}{|l|}{ Number of chronic conditions } \\
\hline 0 & 1.00 & 1.00 & 1.00 \\
\hline 1 & $0.89(0.81-0.97)$ & $0.94(0.85-1.05)$ & $0.92(0.82-1.03)$ \\
\hline$\geq 2$ & $0.98(0.81-1.18)$ & $1.07(0.89-1.28)$ & $1(0.80-1.25)$ \\
\hline
\end{tabular}

Only patients who logged into MyChart were included in these models. Note that these are odds ratio for the odds of not performing an activity given an individual had logged in. These odds were modeled as part of individual zero-inflated maximum-likelihood Poisson models to estimate both (1) the odds of not performing an activity and (2) the number of each of the 3 activities. Bold values are significant at $P<.05$.

FPL, federal poverty level. 